\title{
The protein quality control system manages plant defense compound synthesis
}

Jacob Pollier ${ }^{1,2^{*}}$, Tessa Moses ${ }^{1,2,3,4^{*}}$, Miguel González-Guzmán ${ }^{1,2,}$, Nathan De Geyter ${ }^{1,2}$, Saskia Lippens ${ }^{5,6}$, Robin Vanden Bossche ${ }^{1,2}$, Peter Marhavý, ${ }^{1,2}$, Anna Kremer ${ }^{5}$, Kris Morreel $^{1,2}$, Christopher J. Guérin ${ }^{5,6}$, Aldo Tava ${ }^{7}$, Wieslaw Oleszek ${ }^{8}$, Johan M. Thevelein ${ }^{3,4}$, Narciso Campos ${ }^{9,10}$, Sofie Goormachtig ${ }^{1,2} \&$ Alain Goossens ${ }^{1,2}$

${ }^{1}$ Department of Plant Systems Biology, VIB, Technologiepark 927, B-9052 Gent, Belgium

${ }^{2}$ Department of Plant Biotechnology and Bioinformatics, Ghent University, Technologiepark 927, B-9052 Gent, Belgium

${ }^{3}$ Laboratory of Molecular Cell Biology, Institute of Botany and Microbiology, KU Leuven, Kasteelpark Arenberg 31, B-3001 Leuven-Heverlee, Belgium

${ }^{4}$ Department of Molecular Microbiology, VIB, Kasteelpark Arenberg 31, B-3001 LeuvenHeverlee, Belgium

${ }^{5}$ Bio Imaging Core, VIB, Technologiepark 927, B-9052 Gent, Belgium

${ }^{6}$ Department for Molecular Biomedical Research, VIB, Technologiepark 927, B-9052 Gent, Belgium

${ }^{7}$ Consiglio per la Ricerca e la Sperimentazione in Agricoltura, Centro di Ricerca per le Produzioni Foraggere e Lattiero Casearie, viale Piacenza 29, 26900 Lodi, Italy

${ }^{8}$ Department of Biochemistry, Institute of Soil Science and Plant Cultivation - State Research Institute, ul. Czartoryskich 8, 24-100 Pulawy, Poland

${ }^{9}$ Departament de Bioquímica i Biologia Molecular, Facultat de Biologia, Universitat de Barcelona, Avda Diagonal 643, Barcelona 08028, Spain.

${ }^{10}$ Departament de Genètica Molecular, Centre de Recerca en Agrigenòmica, Consorci CSICIRTA-UAB-UB, Campus UAB, Bellaterra, Barcelona 08193, Spain 
*These authors contributed equally to this work.

'Present address: Instituto de Biología Molecular y Celular de Plantas, Consejo Superior de Investigaciones Cientificas-Universidad Politécnica de Valencia, Ingeniero Fausto Elio s/n, 46022 Valencia, Spain

Jasmonates (JAs) are ubiquitous oxylipin-derived phytohormones that are essential in the regulation of multiple plant processes, encompassing development, growth and defense. Across the plant kingdom, JAs act as elicitors of the production of bioactive secondary metabolites that serve in the defense against attackers ${ }^{1-3}$. Knowledge on the conserved JA perception and early signaling machineries is increasing ${ }^{3-6}$ but the downstream mechanisms that regulate defense metabolism remain largely unknown. Here we show that in the model legume Medicago truncatula JA recruits the endoplasmic reticulum-associated degradation (ERAD) quality control system to manage the production of triterpene saponins, widespread bioactive compounds that share a biogenic origin with sterols ${ }^{7-9}$. An ERAD-type RING membrane-anchor (RMA) E3 ubiquitin (Ub)-ligase is co-expressed with saponin synthesis enzymes to control 3hydroxy-3-methylglutaryl-CoA reductase (HMGR), the rate-limiting enzyme in the supply of the ubiquitous terpene precursor isopentenyl diphosphate. Thereby unrestrained bioactive saponin accumulation is prevented and plant development and integrity secured. This control apparatus is equivalent to the ERAD system that regulates sterol synthesis in yeasts and mammals but that employs distinct E3 Ub-ligases, of the HMGR Degradation 1 (HRD1)-type, to direct destruction of HMGR ${ }^{10-13}$. Hence, the general principles for management of sterol and triterpene saponin biosynthesis are conserved across eukaryotes but can be controlled by divergent regulatory cues. 
To identify regulators of plant triterpene synthesis, we monitored the transcriptome of suspension-cultured $M$. truncatula cells, known to accumulate saponins following elicitation with $\mathrm{JAs}^{14}$. The expression of 8,462 transcripts was visualized by cDNA-AFLP transcript profiling and 282 Methyl JA (MeJA)-responsive tags were identified. The comparable MeJAinduced expression pattern of the genes encoding HMGR, squalene synthase, squalene epoxidase, $\beta$-amyrin synthase (BAS) and CYP93E2, enzymes catalyzing steps in triterpene saponin biosynthesis ${ }^{7-9}$, indicated co-regulation (Fig. 1a and Extended Data Fig. 1-2). Several genes corresponding to potential regulatory factors had maximal transcriptional upregulation prior to or concurrent with that of the triterpene saponin genes, including a MYC-like bHLH protein and the JAZ repressor proteins, known elements of the core JA signaling module ${ }^{4-6}$, as well as a gene (MT067) corresponding to an RMA-like E3 Ub-ligase ${ }^{10}$, denominated MAKIBISHI1 (MKB1) (Extended Data Fig. 3). The early MeJA response of $M K B 1$ was confirmed in the M. truncatula Gene Expression Atlas (MtGEA; http://bioinfo.noble.org/gene$\left.\underline{\text { atlas}^{15}}\right)^{15}$ (Fig. 1b).

To assess MKB1 function, we generated transgenic $M$. truncatula hairy roots in which $M K B 1$ was overexpressed (MKB1 ${ }^{\mathrm{OE}}$ roots) or silenced $\left(\mathrm{MKB} 1^{\mathrm{KD}}\right.$ roots) (Extended Data Fig. 4a). $M K B 1^{\mathrm{KD}}$ roots showed a striking phenotype, in particular when transferred to liquid medium, which caused 'dissociation' of the $\mathrm{MKB} 1^{\mathrm{KD}}$ roots into 'caltrop-like' structures (Fig. 2a and Extended Data Fig. 4b), hence the name 'makibishi', Japanese for caltrop. No such phenotypes were observed in control or $\mathrm{MKB} 1^{\mathrm{OE}}$ roots (Fig. 2b and Extended Data Fig. 4b). Microscopic analysis revealed severe morphological deficiencies in $\mathrm{MKB} 1^{\mathrm{KD}}$ roots (Fig. 2bc), which resembled the defects of oat (Avena sativa) mutants that accumulate incompletely glycosylated forms of the avenacin saponins ${ }^{16}$. Serial block face scanning electron microscopy (SBF-SEM) indicated that cells had an irregular instead of the normal cylindrical shape and that intercellular spaces, typical for the root cortex zone, were completely absent in 
$\mathrm{MKB} 1^{\mathrm{KD}}$ roots (Fig. 2d-e). This might account for the 'makibishi' phenotype since the severe cell enlacing might render the tissue too rigid and lead to ruptures during root development.

To verify possible correlation with altered metabolism, we performed metabolite profiling by liquid chromatography electrospray ionization Fourier transform ion cyclotron resonance mass spectrometry (LC-ESI-FT-ICR-MS $)^{17} \cdot \mathrm{MKB}^{\mathrm{KD}}$ roots were clearly different from control roots, whereas $\mathrm{MKB} 1^{\mathrm{OE}}$ roots showed no significant differences (Fig. 3a; Extended Data Fig. 4c-d). Tens of compounds showed a significantly higher or lower accumulation in $\mathrm{MKB} 1^{\mathrm{KD}}$ roots, among which numerous triterpene saponins, one of the main classes of metabolites known to accumulate in $M$. truncatula $^{18}$. The majority of the upregulated saponins were monoglycosylated compounds, such as 3-O-Glc-medicagenic acid, whereas only higher glycosylated forms, such as soyasaponin I, were represented within the down-regulated saponins (Fig. 3b-c; Extended Data Fig. 4e-f and Extended Data Table 1). Analysis of the growth medium of $\mathrm{MKB} 1^{\mathrm{KD}}$ roots revealed the presence of tens of compounds, including the monoglycosylated saponins, whereas in the growth medium of control roots no metabolites were detected (Extended Data Fig. 5a), indicating release of compounds from the $\mathrm{MKB} 1^{\mathrm{KD}}$ roots. Notably, application of aliquots of $\mathrm{MKB} 1^{\mathrm{KD}}$ root culture medium to control roots caused transient tissue loosening (Extended Data Fig. 5b), suggesting that ectopic accumulation of bioactive monoglycosylated saponins ${ }^{7-9,19}$ and/or other metabolites contributes to the makibishi phenotype and is not a mere consequence of the root defects. These findings suggest that silencing of $M K B 1$ interferes with the biosynthesis of triterpene saponins, which leads to the overaccumulation and release of incompletely glycosylated saponins and correlates with the manifestation of the makibishi phenotype.

Remarkably, the expression of all hitherto known specific saponin biosynthetic genes (BAS, CYP93E2, CYP716A12, UGT73F3 and UGT73K1) was strongly downregulated in $\mathrm{MKB1}^{\mathrm{KD}}$ roots (Fig. 1c and Extended Data Fig. 2). Reduction of expression of some, but not 
all of the genes corresponding to enzymes catalyzing triterpene synthesis up to the oxidosqualene precursor was also observed. Downregulation was not observed with the putative M. truncatula orthologs of genes corresponding to Arabidopsis thaliana sterol biosynthesis enzymes (Extended Data Fig. 6a and b), indicating that $M K B 1$ silencing did not affect transcriptional regulation of triterpene biosynthesis in general. Correspondingly, no $\mathrm{MKB} 1^{\mathrm{KD}}$-specific differences in sterol levels were detected (Extended Data Fig. 6c). Expression of none of the triterpene pathway genes decreased in $\mathrm{MKB}^{\mathrm{OE}}$ roots (Fig. 1c and Extended Data Fig. 6b), in accordance with the lack of an observable phenotype. These findings point towards the occurrence of a saponin-specific feedback mechanism, likely required for coping with the ectopic accumulation of bioactive monoglycosylated saponins. Verification of the expression patterns of GFP-reporter constructs, driven by promoters of the $B A S$ and $U G T 73 K 1$ genes, showed that these genes are ubiquitously expressed in $M$. truncatula hairy roots (Fig. 1d-e), thus excluding that the decrease in their transcript levels is attributable to the developmental defects caused by the makibishi phenotype.

The MKB1 protein contains an N-terminal C3HC4-type RING domain and a C-terminal membrane anchor. Accordingly, MKB1 possesses self-ubiquitination activity in vitro and GFP-tagged MKB1 proteins are visible in an ER-reminiscent network pattern in bombarded onion (Allium cepa) cells and co-localize with a known ER protein in yeast (Extended Data Fig. 7a-d). Thus, MKB1 corresponds to an active, ER-localized E3 Ub-ligase, like its mammalian counterparts, which are involved in $\mathrm{ERAD}^{10}$.

Besides protein quality, the ERAD system also controls sterol synthesis in yeasts and mammals through the regulation of HMGR levels. Yeast does not possess RMA-type proteins and, as mammals, employs the HRD1-type of ERAD E3 Ub-ligases for sterol control ${ }^{10-13}$. Despite this, the lack of sequence similarity between the different types of E3 Ub-ligases and the different membrane topology of the HMGR enzymes from plants, yeasts and mammals 
(Extended Data Fig. 1b-d), we reasoned that MKB1 might survey triterpene saponin synthesis in M. truncatula by targeting HMGR (Extended Data Fig. 1a). In support of this hypothesis, we observed high expression correlation between several of the M. truncatula HMGR genes and MKB1 but not the putative M. truncatula homolog of yeast HRD1 (Fig. 1b). Therefore, we checked accumulation of HMGR proteins in M. truncatula roots by immunoblot analysis with polyclonal antibodies raised against the conserved catalytic domain of Arabidopsis or melon (Cucumis melo) HMGR proteins ${ }^{20,21}$. Only a small increase in HMGR protein levels was detected in $\mathrm{MKB}^{\mathrm{KD}}$ roots as compared to control roots (Extended Data Fig. 8a). Furthermore, we observed that in control roots MeJA application enhanced HMGR transcript levels, whereas HMGR protein levels remained stable (Fig. 4a-b). In MKB1 ${ }^{\mathrm{KD}}$ roots however, also HMGR protein levels increased after MeJA application (Fig. 4b), supporting a role of MKB1 in the control of HMGR levels. Unexpectedly however, HMGR activity was markedly lower in $\mathrm{MKB}^{\mathrm{KD}}$ than control roots (Extended Data Fig. 8b). Analogous to the effect on the saponin-specific transcripts, we speculate that this is caused by post-translational negative control triggered by the ectopic accumulation of bioactive saponins. A similar inverse correlation between HMGR activity and triterpene levels was observed in transgenic Taraxacum brevicorniculatum plants with silenced rubber synthesis, which was postulated to reflect feedback inhibition from oxidosqualene-derived products or precursors ${ }^{22}$. Multilevel control of HMGR has been well documented in yeast and mammals ${ }^{10-12}$, has also been reported in plants ${ }^{20,23}$, and may, for instance, involve phosphorylation.

The relation between MKB1 and HMGR proteins was further examined by three sets of experiments. First, immunoprecipitation assays with tagged MKB1 proteins expressed in $M$. truncatula hairy roots indicated that MKB1 and HMGR proteins can physically associate (Extended Data Fig. S7e), though likely in an indirect manner since yeast-two-hybrid analysis failed to demonstrate direct interaction between MKB1 and M. truncatula HMGR proteins. 
This parallels the situation in yeasts and mammals, where HMGR proteins do not directly interact with HRD-type ERAD Ub-ligases but require INSIG-type proteins as mediators ${ }^{10-13}$. Second, tagging of firefly luciferase with particular M. truncatula HMGR isoforms (HMGR1 and HMGR3) converted it into a target of MKB1-mediated protein degradation in transfected tobacco (Nicotiana tabacum) protoplasts (Extended Data Fig. 8c). Third, we generated transgenic M. truncatula hairy roots overexpressing truncated HMGR4 proteins (tHMGR $4{ }^{\mathrm{OE}}$ roots), devoid of membrane-spanning domains and localizing to the cytosol, by which they are known to be liberated from ERAD control ${ }^{10-13}$. tHMGR $^{\mathrm{OE}}$ roots exhibited a makibishilike phenotype, accumulated monoglycosylated saponins and showed downregulation of saponin gene expression (Fig. 4c-d and Extended Data Fig. 9a-d, f-h), demonstrating that loss of MKB1 activity and expression of 'deregulated' HMGR4 proteins cause similar effects. No good-growing tHMGR $1^{\mathrm{OE}}$ lines could be generated; hence we could not determine whether this effect is unique to the HMGR4 isoform. Blocking HMGR activity in control roots by lovastatin treatment caused growth inhibition but did not mimic the makibishi phenotype (Extended Data Fig. 9e), confirming that mere loss of HMGR activity cannot account for the $\mathrm{MKB} 1^{\mathrm{KD}}$ effects.

Finally, we demonstrated that MKB1 can target yeast HMGR and thereby complement a yeast strain devoid of HRD1, despite the lack of sequence and topology similarity between both the E3 Ub-ligases and their native targets (Fig. 4e; Extended Data Fig. 9i and Extended Data Fig. 1). Hence, although M. truncatula uses an ERAD system different from the one that directs sterol-regulated destruction of HMGR enzymes in yeast and mammals, it appears compatible. Mammals and yeasts use sterols or IPP-derived non-sterols to regulate HMGR ${ }^{24,25}$. The divergent sequences of the plant proteins might have allowed the evolution of a plantspecific gateway to the control of HMGR activity, e.g. regulated by specific saponin intermediates or involving plant-specific 'mediator' proteins (the INSIG-proteins that are 
conserved between yeast and mammalians are not present in plants). Identifying these elements will be key to unravel the molecular mechanisms that control plant HMGR activity. Possibly MKB1 may manage more ER-localized proteins involved with saponin synthesis, such as cytochrome P450 enzymes, chaperones, or regulators. In this way the plant guarantees self-protection from its own weapons and safeguards development and integrity when trying to eliminate attackers. 


\section{METHODS SUMMARY}

Generation of DNA constructs. Standard molecular biology protocols and Gateway (Invitrogen) technology were followed to obtain expression clones.

Generation and profiling of transgenic $M$. truncatula hairy roots. Transgenic $M$. truncatula (ecotype Jemalong J5) hairy roots were created by A. rhizogenes-mediated transformation ${ }^{17}$. Elicitation, microscopy analysis, and transcript and metabolite profiling were performed as described ${ }^{14,17,26,27}$ with modifications.

MKB1 and HMGR assays. Standard molecular biology protocols were followed to assess the localization and ubiquitination activity of MKB1. HMGR stability and activity were assessed in stable transformed M. truncatula hairy roots and in transiently transfected tobacco

protoplasts as described previously ${ }^{20,28}$, with modifications. Immunoprecipitation with tagged M. truncatula proteins was performed according to a method developed for Arabidopsis $\operatorname{proteins}^{29}$.

Full Methods and any associated references are available in the online version of the paper at www.nature.com/nature.

\section{References}

1. Wasternack, C. Jasmonates: an update on biosynthesis, signal transduction and action in plant stress response, growth and development. Ann. Bot. 100, 681-697 (2007).

2. Pauwels, L., Inzé, D. \& Goossens, A. Jasmonate-inducible gene: what does it mean? Trends Plant Sci. 14, 87-91 (2009). 
3. De Geyter N., Gholami A., Goormachtig S., \& Goossens A. Transcriptional machineries in jasmonate-elicited plant secondary metabolism. Trends Plant Sci. 17, 349-359 (2012).

4. Browse, J. Jasmonate passes muster: a receptor and targets for the defense hormone. Annu. Rev. Plant Biol. 60, 183-205 (2009).

5. Memelink, J. Regulation of gene expression by jasmonate hormones. Phytochemistry $\mathbf{7 0}$, 1560-1570 (2009).

6. Pauwels, L. \& Goossens, A. The JAZ proteins: a crucial interface in the jasmonate signaling cascade. Plant Cell 23, 3089-3100 (2011).

7. Osbourn, A., Goss, R. J. \& Field, R. A. The saponins - polar isoprenoids with important and diverse biological activities. Nat. Prod. Rep. 28, 1261-1268 (2011).

8. Augustin, J. M., Kuzina, V., Andersen, S. B. \& Bak, S. Molecular activities, biosynthesis and evolution of triterpenoid saponins. Phytochemistry 72, 435-457 (2011).

9. Moses, T., Pollier, J., Thevelein, J.M. \& Goossens, A. Bioengineering of plant (tri)terpenoids: from metabolic engineering of plants to synthetic biology in vivo and in vitro. New Phytol., in press, doi: 10.1111/nph.12325.

10. Hirsch, C., Gauss, R., Horn, S. C., Neuber, O. \& Sommer, T. The ubiquitylation machinery of the endoplasmic reticulum. Nature 458, 453-460 (2009).

11. Hampton, R. Y. \& Garz R. M. Protein quality control as a strategy for cellular regulation: lessons from ubiquitin-mediated regulation of the sterol pathway. Chem. Rev. 109, 15611574 (2009).

12. Jo, Y. \& DeBose-Boyd, R. A. Control of cholesterol synthesis through regulated ERassociated degradation of HMG CoA reductase. Crit. Rev. Biochem. Mol. Biol. 45, $185-$ 198 (2010).

13. Burg, J. S. \& Espenshade, P. J. Regulation of HMG-CoA reductase in mammals and yeast. Prog. Lipid Res. 50, 403-410 (2011).

14. Suzuki, H., Achnine, L., Xu, R., Matsuda, S. P. T. \& Dixon, R. A. A genomics approach to the early stages of triterpene saponin biosynthesis in Medicago truncatula. Plant J. 32, 1033-1048 (2002).

15. He, J. et al. The Medicago truncatula gene expression atlas web server. $B M C$ Bioinformatics 10, 441 (2009).

16. Mylona, P. et al. Sad3 and sad4 are required for saponin biosynthesis and root development in oat. Plant Cell 20, 201-212 (2008). 
17. Pollier, J., Morreel, K., Geelen, D. \& Goossens, A. Metabolite profiling of triterpene saponins in Medicago truncatula hairy roots by liquid chromatography Fourier transform ion cyclotron resonance mass spectrometry. J. Nat. Prod. 74, 1462-1476 (2011).

18. Dixon, R. A. \& Sumner, L. W. Legume natural products: understanding and manipulating complex pathways for human and animal health. Plant Physiol. 131, 878-885 (2003).

19. Oleszek, W. Structural specificity of alfalfa (Medicago sativa) saponin haemolysis and its impact on two haemolysis-based quantification methods. J. Sci. Food Agric. 53, 477-485 (1990).

20. Leivar, P. et al. Multilevel control of Arabidopsis 3-hydroxy-3-methylglutaryl coenzyme A reductase by protein phosphatase 2A. Plant Cell 23, 1494-1511 (2011).

21. Kobayashi, T., Kato-Emori, S., Tomita, K. \& Ezura, H. Detection of 3-hydroxy-3methylglutaryl-coenzyme A reductase protein Cm-HMGR during fruit development in melon (Cucumis melo L.). Theor. Appl. Genet. 104, 779-785 (2002).

22. Post, J. et al. Laticifer-specific cis-prenyltransferase silencing affects the rubber, triterpene, and inulin content of Taraxacum brevicorniculatum. Plant Physiol. 158, 14061417 (2012).

23. Hemmerlin, A. Post-translational events and modifications regulating plant enzymes involved in isoprenoid precursor biosynthesis. Plant Sci. 203-204, 41-54 (2013).

24. Sever, N. et al. Insig-dependent ubiquitination and degradation of mammalian 3-hydroxy3-methylglutaryl-CoA reductase stimulated by sterols and geranylgeraniol. J. Biol. Chem. 278, 52479-52490 (2003).

25. Garza, R. M., Tran, P. N. \& Hampton, R. Y. Geranylgeranyl pyrophosphate is a potent regulator of HRD-dependent 3-hydroxy-3-methylglutaryl-CoA reductase degradation in yeast. J. Biol. Chem. 284, 35368-35380 (2009).

26. Vuylsteke, M., Peleman, J. D. \& van Eijk, M. J. T. AFLP-based transcript profiling (cDNA-AFLP) for genome-wide expression analysis. Nat. Protoc. 2, 1399-1413 (2007).

27. Wilke, S.A. et al. Deconstructing complexity: serial block-face electron microscopic analysis of the hippocampal mossy fiber synapse. J. Neurosci. 33, 507-522 (2013).

28. De Sutter, V. et al. Exploration of jasmonate signalling via automated and standardized transient expression assays in tobacco cells. Plant J. 44, 1065-1076 (2005).

29. Bassard, J.E. et al. Protein-protein and protein-membrane associations in the lignin pathway. Plant Cell 24, 4465-4482 (2012). 
Supplementary Information is linked to the online version of the paper at www.nature.com/nature.

Acknowledgements. We thank Wilson Ardiles-Diaz for sequencing the cDNA-AFLP tags, Renaat Dasseville, Riet De Rycke, Jan Geerinck, Lander Ingelbrecht, Sophie Carbonelle, Kristiina Himanen and Laurens Pauwels, for excellent technical advice and assistance, Tom Beeckman for helpful discussion, Annick Bleys for help in preparing the manuscript, Richard Dixon (Samuel Roberts Noble Foundation, Ardmore, USA) for providing the M. truncatula cell line, Hiroshi Ezura (University of Tsukuba, Japan) for providing the melon antibody, and Randy Hampton (UC San Diego, USA), Alexandra Stolz and Dieter Wolf (Universitaet Stuttgart, Germany) for providing yeast strains, respectively. The research leading to these results has received funding from the Agency for Innovation by Science and Technology in Flanders (“Strategisch Basisonderzoek" Combiplan project SBO040093), the European Union Seventh Framework Programme FP7/2007-2013 under grant agreement number 222716 SMARTCELL and the Spanish Ministerio de Economía y Competitividad under grant BFU2011-24208. T.M. and N.D.G. are indebted to the VIB International PhD Fellowship Program and the Agency for Innovation by Science and Technology for predoctoral fellowships, respectively. J.P. and S.L. are postdoctoral fellows of the Research Foundation Flanders (FWO).

Author Contributions. J.P., T.M., M.G.-G., N.D.G., S.L., R.V.B., P.M., A.K., C.G.J., A.T., W.O., N.C. and A.G. performed experiments and analyzed the results. J.P., T.M., M.G.-G., N.D.G., S.L., K.M., C.G.J., S.G., N.C. and A.G. designed experiments and analyses. J.P., T.M., J.M.T. and A.G. wrote the manuscript. All authors commented on the results and the manuscript. 
Author Information. The GenBank accession number for MKB1 is JF714982. Reprints and permissions information is available at www.nature.com/reprints. The authors declare no competing financial interests. Correspondence and requests for materials should be addressed to A.G. (alain.goossens@psb.vib-ugent.be). 


\section{Figure legends}

\section{Figure 1| Expression of $M K B 1$ and saponin synthesis genes is correlated.}

a, Subcluster of the M. truncatula transcriptome with genes involved in triterpene biosynthesis or JA signaling. Treatments and time points (in h) are indicated at the top. Blue and yellow boxes reflect transcriptional activation and repression relative to the average expression level, respectively. b, Coexpression analysis of $M K B 1$ (blue), HMGR1 (red), HMGR4 (orange), and HRD1 (black) in M. truncatula roots and suspension cells with the MtGEA software ${ }^{15}$. Arrows, expression in MeJA treated suspension cells. c, qRT-PCR analysis of saponin genes in control $(\mathrm{CTR}), \mathrm{MKB}^{\mathrm{OE}}(\mathrm{OE})$ and $\mathrm{MKB} 1^{\mathrm{KD}}(\mathrm{KD})$ roots. $\mathrm{Y}$-axis, expression ratio relative to the normalized transcript levels of control line 3 in log scale. Error bars, standard error of the mean $(\mathrm{n}=3)$. Statistical significance was determined by Student's $t$ test (* $\mathrm{P}<0.1, * * \mathrm{P}<0.01, * * * \mathrm{P}<0.001)$. d, e, Fluorescence (d) and confocal (e) microscopy analysis of cell-specific expression from $p B A S$ - and $p U G T 73 K 1-G F P$ constructs in $M$. truncatula hairy roots.

\section{Figure 2| MKB1 silencing causes the 'makibishi’ phenotype.}

a, Fluorescence microscopy, b, confocal microscopy and c, scanning electron microscopy analysis of $\mathrm{CTR}, \mathrm{MKB} 1^{\mathrm{OE}}$, and $\mathrm{MKB} 1^{\mathrm{KD}}$ roots grown in liquid medium. d, e, 3D SEM image stacks visualizing the cell structures of $\mathrm{CTR}$ and $\mathrm{MKB} 1^{\mathrm{KD}}$ roots grown on solid medium. IMOD, FIJI and Ilastik software were used to generate orthogonal slices (d) and 3D reconstructions (e). Yellow lines indicate positions of the corresponding orthogonal views. Red arrows indicate the intercellular spaces in CTR roots. Bar, $10 \mu \mathrm{m}$.

Figure 3| MKB1 silencing causes ectopic accumulation of monoglycosylated saponins. 
a, LC-ESI-FT-ICR-MS chromatograms of an extract from CTR (black) and MKB1 ${ }^{\mathrm{KD}}$ (red) roots. The peak at $t_{\mathrm{R}} 26.71 \mathrm{~min}$ represents $3-O$-Glc-medicagenic acid. b, S-plot derived from partial least squares discriminant analysis (PLS-DA). Metabolites in the lower left and upper right quadrants (marked by dotted red lines) are significantly higher and lower abundant in the $\mathrm{MKB} 1^{\mathrm{KD}}$ samples, respectively. c, Average total ion current (TIC) of the peaks colored in red in the S-plot and corresponding to soyasaponin I (upper) and 3-O-Glc-medicagenic acid (lower). Error bars indicate the standard error $(n=4)$.

\section{Figure 4| MKB1 targets the HMGR enzyme.}

a, b, HMGR expression in mock- or MeJA-treated CTR and $\mathrm{MKB1}{ }^{\mathrm{KD}}$ roots. a, qRT-PCR analysis of $H M G R$ transcript levels. b, Immunoblot analysis of HMGR protein levels. The Yaxes show the ratio relative to the normalized levels of the mock at $0 \mathrm{~h}$. Error bars indicate the standard error of the mean ( $\mathrm{n}=3$ and 2, respectively). c, Fluorescence microscopy analysis of $\mathrm{CTR}, \mathrm{MKB} 1^{\mathrm{KD}}$, and $\mathrm{tHMGR} 4^{\mathrm{OE}}$ roots grown in liquid medium. d, Principal component analysis of samples from $\mathrm{MKB} 1^{\mathrm{KD}}$ (red), $\mathrm{tHMGR} 4^{\mathrm{OE}}$ (blue) and CTR (black) roots. e, HRD1 $(\mathrm{H})$ or $h r d 1(\mathrm{~h})$ yeasts were transformed with $M K B 1(+\mathrm{M})$ or a ligase-dead version $(+\mathrm{m})$, spotted in a 10-fold dilution series on selective SD medium supplemented (LOV) or not $(\mathrm{CON})$ with lovastatin and grown for 2 days at $30^{\circ} \mathrm{C}$. The empty vector pAG426GPD was used as a control (-). Left and right panels show complementation in the RHY400(H)/RHY401(h) and YWO1167(H)/YWO1528(h) genotypes, respectively. 


\section{METHODS}

\section{M. truncatula suspension cell culture maintenance and elicitation}

M. truncatula cell cultures (kindly provided by Richard Dixon, Ardmore, USA), were maintained as described ${ }^{14}$. For elicitation, 7 days after inoculation of $75 \mathrm{~mL}$ of a 14-day-old suspension culture into $175 \mathrm{~mL}$ fresh medium, cells were treated with $100 \mu \mathrm{M}$ MeJA or an equivalent amount of the solvent ethanol as a control. Samples were harvested, vacuum filtrated, and frozen at $-80^{\circ} \mathrm{C}$.

\section{Transcript profiling}

Total RNA from M. truncatula cells was prepared with TRIZol (Invitrogen, Carlsbad, CA) and reverse transcribed to double-stranded cDNA as described ${ }^{26}$. After appropriate sample preparation, cDNA-AFLP based transcript profiling was done with all 128 possible $B s t \mathrm{YI}+1 / M s e \mathrm{I}+2$ primer combinations ${ }^{26}$. Gel image analysis, quantification of band intensities, selection of differentially expressed gene tags, cluster analysis, sequencing, and BLAST analysis were carried out as described ${ }^{26,30}$.

For quantitative Real Time PCR (qRT-PCR), total RNA was extracted with the RNeasy mini kit (Qiagen), and cDNA prepared with SuperScript ${ }^{\mathrm{TM}}$ II Reverse Transcriptase (Invitrogen). Primers were designed with Beacon Designer version 4.0 (Premier Biosoft International, Palo Alto, CA, USA). qRT-PCR was carried out with a Lightcycler 480 (Roche) and SYBR Green QPCR Master Mix (Stratagene). For reference genes, 40S ribosomal protein S8 (40S) (TC160725 of the MTGI from TIGR) and translation elongation factor $1 \alpha$ (ELF1 $\alpha)$ 
(TC148782 of the MTGI from TIGR) were used. Reactions were done in triplicate and for the relative quantification with multiple reference genes qBase was used ${ }^{31}$.

\section{Generation of DNA constructs}

For silencing by means of hairpin RNAi, the 471-bp $M K B 1$ cDNA-AFLP fragment was PCRamplified and by Gateway ${ }^{\mathrm{TM}}$ recombination cloned into the binary vector pK7GWIWG2D(II) ${ }^{32}$. The resulting expression clone was transformed into the Agrobacterium rhizogenes strain LBA 9402/12 for generation of hairy roots.

To identify the full-length open reading frame (FL-ORF) of $M K B 1$, the cDNA-AFLP tag sequence was used for a BLASTN search against the Medicago truncatula Gene Index database (http://compbio.dfci.harvard.edu/tgi/). The MKB1 FL-ORF consensus sequence (TC149901; GenBank accession JF714982), the M. truncatula HMGR1, HMGR2, HMGR3, HMGR4, and HMGR5 sequences (GenBank accession EU302813, EU302814, EU302815, EU302816, and

EU302817, respectively $)^{33}$ and the sequences of the $M$. truncatula homologs of Arabidopsis JAZ1 and CKS1 (GenBank accession XM_003595306 and XM_003606264, respectively) were PCR-amplified and by Gateway ${ }^{\mathrm{TM}}$ recombination cloned into the entry vector pDONR221. To obtain entry clones with and without stop codon, Gateway primers were designed according to Underwood et al. ${ }^{34}$. The $M K B 1$ and HMGR4 entry vectors were used as a template to amplify truncated versions of the ORFs, as well as to create point mutations with the GeneTailor SiteDirected Mutagenesis system (Invitrogen).

The promoter sequences of $B A S$ and $U G T 73 K 1$ were retrieved from the from the $M$. truncatula genome v3.5 ${ }^{35}$ (Medtr4g005190 and Medtr4g031800, respectively). For both promoters, 1000 bases upstream of the start codon were PCR-amplified and by Gateway ${ }^{\mathrm{TM}}$ recombination cloned into the entry vector pDONR221. 
All entry constructs were sequence-verified. For stable overexpression experiments, Gateway recombination was carried out with the pK7WG2D binary vector ${ }^{32}$, and the resulting clone transformed to A. rhizogenes. For transient overexpression in tobacco protoplasts, the ORFs were fused at their C-terminus with the firefly luciferase ORF by a fusion PCR and Gateway recombined in the p2GW7 vector ${ }^{32}$. For localization experiments in onion cells, Gateway recombination was carried out with the $\mathrm{pK} 7 \mathrm{WGF} 2$ vector $^{32}$. For recombinant protein production, the sequences were recombined in the pDEST15 expression vector, and the resulting clones transformed to E. coli BL21 (DE3) cells. For the yeast complementation and localization assays, the pAG426GPD vector ${ }^{36}$ was used as the destination vector. To generate bait proteins for immunoprecipitation, the ORFs were fused either $\mathrm{N}$ - or C-terminally to the protein G-Streptavidin (GS) tag by Gateway recombination as described ${ }^{37}$. For promoter analysis, the $B A S$ and $U G T 73 K 1$ promoter sequences were put in front of a fusion of the GFP and $\beta$-glucuronidase (GUS) coding sequences in the pKGWFS7 vector ${ }^{32}$.

\section{Phylogenetic analysis}

The protein sequences were aligned with ClustalW and the resulting alignments were

manually adjusted. The phylogenetic tree was generated in MEGA 4.0.1 software ${ }^{38}$, by the Neighbor-Joining method, and bootstrapping was done with 10,000 replicates. The evolutionary distances were computed with the Poisson correction method, and all positions containing gaps and missing data were eliminated from the data set (complete deletion option).

\section{Generation and phenotypic analysis of transgenic $M$. truncatula hairy roots}


A. rhizogenes-mediated transformation and cultivation of M. truncatula (ecotype Jemalong J5) hairy roots was done according to Pollier et al. ${ }^{17}$.

Samples for scanning electron microscopy (SEM) were prepared as described ${ }^{39}$. Briefly, after the first fixation step in $4 \%$ paraformaldehyde, $1 \%$ glutaraldehyde in $2 \mathrm{mM}$ sodium phosphate buffer, the root samples were fixed in $1 \%$ osmium tetroxide solution (Fluka) for 2 hours, and subsequently subjected to a dehydration series to $100 \%$ ethanol. Next, the root samples were critical-point dried and sputter-coated with gold particles before they were examined with a JEOL JSM-5600 LV or Zeiss Auriga SEM microscope under an acceleration voltage of $10 \mathrm{kV}$ or $1.5 \mathrm{kV}$, respectively.

For SBF-SEM, plant roots were fixed in $0.15 \mathrm{M}$ cacodylate $\mathrm{pH} 7.4,2.5 \%$ glutaraldehyde (EMS, Hatfield, USA) and 2\% paraformaldehyde (AppliChem, Darmstadt, Germany) for $2 \mathrm{~h}$. To protect the specimens against mechanical stress, the root tips were dipped in $0.6 \%(\mathrm{w} / \mathrm{v})$ low melting agarose (Sigma) in PBS. Samples were transferred to fresh fixative and kept overnight at $4^{\circ} \mathrm{C}$. The next day, samples were washed 5 times for $3 \mathrm{~min}$ in cold $0.15 \mathrm{M}$ cacodylate buffer. En bloc contrast staining was performed by consecutive incubations in heavy metal containing solutions. Between these steps samples were washed 5 times for $3 \mathrm{~min}$ in ultra-pure water (UPW). The first staining step was 1 -hour incubation on ice in $1.5 \%$ potassium ferrocyanide and $2 \%$ aqueous osmium tetroxide in $0.15 \mathrm{M}$ cacodylate buffer. After washing, the samples were incubated for $20 \mathrm{~min}$ in a fresh thiocarbohydrazide solution (Sigma) (1\% w/v in UPW) at room temperature (RT). The next wash step was followed by overnight incubation in $2 \%$ osmium in UPW at RT for 30 min and $2 \%$ uranyl acetate (EMS) at $4{ }^{\circ} \mathrm{C}$. The following day, Walton's lead aspartate staining was performed for $30 \mathrm{~min}$ at $60^{\circ} \mathrm{C}$. For this, a $30-\mathrm{mM} \mathrm{L}$ aspartic acid solution was used to freshly dissolve lead nitrate (Sigma) (20 mM, pH 5.5). The solution was filtered after $30 \mathrm{~min}$ incubation at $60^{\circ} \mathrm{C}$. After final washing steps the samples were dehydrated using ice-cold solutions of 70\%, 90\%, 100\% ethanol (anhydrous), 10 min 
each. Resin embedding was done using Durcupan AMC (EMS) by first placing the samples in $50 \%$ ethanol/Durcupan overnight, followed by 2 incubations in 100\% Durcupan ( 8 hours and overnight). The next day samples were put in fresh Durcupan solution and placed at $60^{\circ} \mathrm{C}$ for 48 hours. For SBF imaging the resin embedded root tips were mounted on an aluminum specimen pin (Gatan, Pleasanton, USA), using conductive epoxy (Circuit Works, Waukegan, USA) and the root tip facing upward. The specimens were trimmed in a pyramid shape using an ultramicrotome and coated with $5 \mathrm{~nm}$ of Pt, in a Quorum sputter coater (Quorum Technologies, West Sussex, UK). The aluminum pins were placed in the Gatan 3View2 in a Zeiss Merlin SEM, for imaging using $1.3 \mathrm{kV}$ and imaged using the Gatan Digiscan II ESB detector. For registration of the 3D image stack, IMOD (http://bio3d.colorado.edu/imod/) was used.

Orthogonal views and linear brightness contrast adjustments were obtained in Fiji (http://fiji.sc/Fiji). For segmentation and isosurface rendering Ilastik 0.5 was used (http://www.ilastik.org). The datasets were automatically segmented using the seeded watershed algorithm.

\section{Metabolite profiling}

M. truncatula hairy roots were grown for 21 days in liquid medium. The hairy roots were harvested and the medium collected from five biological repeats of three independent transgenic lines per transgene construct. Processing and metabolite extraction from hairy root tissue was performed as described ${ }^{17}$.

To remove salts from the samples of the culture medium, $1 \mathrm{~mL}$ of medium was brought on a $100 \mathrm{mg}$ Extract-Clean ${ }^{\mathrm{TM}}$ SPE column (Mandel, Guelph, Ontario, Canada) preconditioned with $1 \mathrm{~mL} 100 \% \mathrm{MeOH}$ and $1 \mathrm{~mL}$ water acidified with $0.1 \%(\mathrm{v} / \mathrm{v})$ acetic acid. After washing 
with $1 \mathrm{~mL}$ acidified water, samples were eluted in $1 \mathrm{~mL}$ methanol. The methanol eluent was evaporated to dryness under vacuum and the residue dissolved in $200 \mu 1$ water for analysis.

LC-ESI-FT-ICR-MS analysis was carried out as described ${ }^{17}$. Briefly, reversed-phase LC was achieved using an Acquity UPLC BEH C18 column $(150 \times 2.1 \mathrm{~mm}, 1.7 \mu \mathrm{m}$; Waters, Milford, MA) coupled to a second Acquity UPLC BEH C18 column (100x2.1 mm, $1.7 \mu \mathrm{m})$. The following gradient using water/acetonitrile (99:1, v/v) (solvent A) and acetonitrile/water $(99: 1, \mathrm{v} / \mathrm{v})($ solvent B), both acidified with $0.1 \%(\mathrm{v} / \mathrm{v})$ acetic acid, was run: time $0 \mathrm{~min}, 5 \% \mathrm{~B}$; $30 \mathrm{~min}, 55 \% \mathrm{~B} ; 35 \mathrm{~min}, 100 \% \mathrm{~B}$. The loop size, flow rate, and column temperature were $25 \mu \mathrm{L}, 300 \mu \mathrm{L} / \mathrm{min}$, and $80^{\circ} \mathrm{C}$, respectively. Negative ionization was obtained using a capillary temperature of $150^{\circ} \mathrm{C}$, sheath gas of 25 (arbitrary units), auxiliary gas of 3 (arbitrary units), and a spray voltage of $4.5 \mathrm{kV}$. Full FT-MS spectra between m/z 120-1400 were recorded at a resolution of 100,000. Full FT-MS scans were interchanged with dependent $\mathrm{MS}^{2}$ scan events, in which the most abundant ion of the previous FT-MS scan was fragmented, and two dependent $\mathrm{MS}^{3}$ scan events in which the two most abundant daughter ions of the $\mathrm{MS}^{2}$ scans were fragmented. The collision energy was set to $35 \%$.

The resulting chromatograms were integrated and aligned with the XCMS package ${ }^{40}$ in $\mathrm{R}$ version 2.6.1. with the following parameter values: $x \operatorname{cms} \operatorname{Set}(f w h m=6, \max =300$, snthresh=2, $\operatorname{mzdiff}=0.1)$, group $(b w=8, \max =300)$, retcor(method=loess, family=symmetric $)$. A second grouping was done with the same parameter values. Due to in-source fragmentation, multiple $m / z$ peaks for each compound were often observed.

The principal component analysis (PCA) and partial least squares discriminant analysis (PLSDA) were performed with the SIMCA-P 11 software package (Umetrics AB, Umeå, Sweden) with Pareto-scaled mass spectrometry data. Peaks with an absolute covariance value above 0.03 and an absolute correlation value above 0.6 were considered as significantly different. 
For identification of the differential metabolites, $\mathrm{MS}^{\mathrm{n}}$ spectra were elucidated as described $^{17,41,42}$. To experimentally validate the annotation of several of the elucidated saponins, representative samples of the medium, $\mathrm{MKB}^{\mathrm{KD}}$ and CTR lines were re-analyzed in the presence of standard saponins ${ }^{43-47}$.

Sterols were extracted from $100 \mathrm{mg}$ of ground roots using methanol, which was dried under vacuum and further extracted with hexane:water (1:1). The hexane phase was dried under vacuum and trimethylsilylated for GC-MS analysis using GC model 6890 and MS model 5973 (Agilent). A 1- $\mu \mathrm{l}$ aliquot was injected in splitless mode into a VF-5ms capillary column (Varian CP9013, Agilent) and operated at a constant helium flow of $1 \mathrm{ml} / \mathrm{min}$. The injector was set to $280^{\circ} \mathrm{C}$ and the oven was programmed at $80^{\circ} \mathrm{C}$ for 1 min post injection, ramped to $280^{\circ} \mathrm{C}$ at $20^{\circ} \mathrm{C} / \mathrm{min}$, held at $280^{\circ} \mathrm{C}$ for $30 \mathrm{~min}$, ramped to $320^{\circ} \mathrm{C}$ at $20^{\circ} \mathrm{C} / \mathrm{min}$, held at $320^{\circ} \mathrm{C}$ for $1 \mathrm{~min}$, and finally cooled to $80^{\circ} \mathrm{C}$ at $50^{\circ} \mathrm{C} / \mathrm{min}$ at the end of the run. The MS transfer line was set to $250^{\circ} \mathrm{C}$, the MS ion source to $230^{\circ} \mathrm{C}$, and the quadrupole to $150^{\circ} \mathrm{C}$, throughout. MS spectra were generated by scanning the $\mathrm{m} / \mathrm{z}$ range of $60-800$ with a solvent delay of $7.8 \mathrm{~min}$. The areas of the peaks were calculated using the default settings of the AMDIS software (v2.6, NIST, USA).

\section{Ubiquitination assay}

Recombinant GST-MKB1 fusion proteins (truncated with or without mutation) were purified according to the manufacturer's instructions with Glutathione Sepharose ${ }^{\mathrm{TM}} 4 \mathrm{~B}$ resin columns (GE Healthcare) from transformed E. coli cells, pretreated for 2 hours with isopropyl- $\beta-D-1-$ thiogalactopyranoside (IPTG). A protein refolding step to assure the full ion $\mathrm{Zn}$ charge of the GST-MKB1 fusion proteins was included by incubation with refolding buffer (20 mM Hepes, 
pH 7.4, $0.02 \mathrm{mM} \mathrm{ZnCl}_{2}, 1.5 \mathrm{mM} \mathrm{MgCl} 2,150 \mathrm{mM} \mathrm{KCl}, 0.2 \mathrm{mM}$ EDTA, 20\% glycerol, $0.05 \%$ Triton $\mathrm{X}-100)$ for 1 hour at $4^{\circ} \mathrm{C}$.

Ubiquitination reactions were done in a total volume of $30 \mu 1$ using $15 \mu 1$ of the refolded GST-MKB1 bound to glutathione resin. The reaction contained $300 \mathrm{ng}$ of GSTMKB1 fusion protein as E3 Ub-ligase, $250 \mathrm{ng}$ of the Ub-activating Enzyme (UBE1) from rabbit (BostonBiochem), $400 \mathrm{ng}$ of human recombinant UbcH5a protein (BostonBiochem), and $2 \mu \mathrm{g}$ of His6-Ub from human (BostonBiochem) in ubiquitination buffer (50 mM Hepes, pH 7.4, 2 mM ATP, $5 \mathrm{mM} \mathrm{MgCl} 2,2 \mathrm{mM}$ DTT, $0.02 \mathrm{mM} \mathrm{ZnCl} 2$ ). The ubiquitination reactions were incubated for 1 hour at $30^{\circ} \mathrm{C}$ and stopped by adding $2 \times$ Laemmli buffer. Samples were resolved on $8 \%$ SDS-PAGE followed by protein immunoblot analysis with RGS/penta/tetra His antibody (Qiagen) and $\alpha$-GST (GE Healthcare) antibodies.

\section{Particle bombardment of onion epidermis cells.}

The constructs for localization were transformed into onion epidermis cells by microparticle bombardment with a PDS-1000/He Biolistic ${ }^{\circledR}$ Particle Delivery System (Bio-Rad Laboratories, Hercules, CA, USA). To this end, $1 \mathrm{mg}$ of 1.6- $\mu \mathrm{m}$ Gold Microcarriers (Bio-Rad Laboratories) was coated with $5 \mu \mathrm{g}$ plasmid DNA according to the manufacturer's instructions. The coated particles were bombarded into onion epidermis slices of approximately $2.5 \times 2.5 \mathrm{~cm}$, placed on solid MS medium (pH 5.8) supplemented with 1\% (w/v) sucrose, with 1100 psi rupture discs and a vacuum of 0.1 bar. Subsequently, the onion slices were stored in the dark for 24 hours at room temperature before analysis by confocal microscopy.

\section{Confocal microscopy}


M. truncatula hairy roots, bombarded onion slices and transformed BY4742 yeast cells (with an integrated RFP tagged Sec13 protein for ER and Golgi marking) ${ }^{48,49}$ were analyzed by confocal microscopy with the FV10 ASW Olympus Confocal with a water immersion $63 \times$ objective.

\section{Analysis of M. truncatula HMGR protein levels and activity}

Protein extraction from M. truncatula hairy roots was carried out as described ${ }^{20}$. Determination of HMGR protein levels by immunoblot analysis with polyclonal antibodies raised against the conserved catalytic domain of Arabidopsis or melon (Cucumis melo) HMGR proteins was performed essentially as decribed ${ }^{20,21}$. Determination of HMGR-specific activity was carried out as described ${ }^{20}$.

\section{Immunoprecipitation assays}

Protein extraction from M. truncatula hairy roots producing GS-tagged bait proteins was carried out according to a protocol described for Arabidopsis cells ${ }^{29}$. Protein purification and precipitation were performed as described $^{37}$ except that precipitation was performed immediately after the elution via the AcTEV digest.

\section{HMGR degradation assays in tobacco protoplasts}

Protoplast preparation from tobacco Bright Yellow-2 cells, automated transfection, lysis and firefly luciferase assays were carried out as described ${ }^{28}$.

\section{Yeast complementation and protein degradation assays}


Two sets of $S$. cerevisiae strains were used for the complementation and protein degradation assays, namely strains YWO1167 (W303 Mat $\alpha$, ura3-1, his3-11,15, leu2-3,112, trp1-1, ade21ocre, can1-100, prc1-1, doa10::KanMX) and its hrd1 (4der3/hrd1::HIS3) knock-out (YWO1528), on the one hand, and RHY400 (Mat a, ade2-101, his34200, lys2-801, met2, hmg1::LYS2, hmg2::HIS3, ura3-52::6MYC-HMG2) expressing 6myc-Hmg2 and its hrd1-1 mutant RHY $401^{50}$, on the other hand. Transformations were carried out with the high-efficiency lithium acetate/single-stranded carrier DNA/polyethylene glycol method. The transformed yeast strains were selected on minimal medium (2.67\% minimal SD Base with $0.077 \%$-Ura DO supplement; Clontech) supplemented with $30 \mathrm{mg} / 1$ adenine and methionine.

For the $h r d 1$ mutant phenotype complementation assays, minimal medium supplemented with $100 \mu \mathrm{g} / \mathrm{ml}$ or $175 \mu \mathrm{g} / \mathrm{ml}$ lovastatin was used for the RHY and YWO strains, respectively. A stock solution of $25 \mathrm{mg} / \mathrm{ml}$ lovastatin was prepared by the hydrolysis of a $100-\mathrm{mg} / \mathrm{ml}$ solution in $95 \%$ ethanol with $1 \mathrm{~N} \mathrm{NaOH}$ at $55^{\circ} \mathrm{C}$ for $40 \mathrm{~min}$, followed by addition of $1 \mathrm{M}$ Tris- $\mathrm{HCl}$ (pH 8.0) and adjustment of $\mathrm{pH}$ to 8.0 with $1 \mathrm{~N} \mathrm{HCl}$.

The 6myc-Hmg2 level was determined by immunoblotting of whole cell protein extracts prepared from yeast cells by washing with minimal medium containing $0.1 \% \mathrm{NaN}_{3}$, followed by resuspension in $100 \mu \mathrm{l}$ of SUTE buffer $(8 \mathrm{M}$ urea, $1 \%$ SDS, $10 \mathrm{mM}$ Tris base, $10 \mathrm{mM}$ EDTA, $\mathrm{pH}$ adjusted to 7.5) containing Complete protease inhibitors (Roche) at $\mathrm{pH}$ 6.8. The cells were lysed by vortexing at high speed with acid-washed $0.5-\mathrm{mm}$ glass beads. The lysate was boiled for $10 \mathrm{~min}$ at $65^{\circ} \mathrm{C}$ after addition of $100 \mu \mathrm{USB}$ buffer ( $8 \mathrm{M}$ urea, $4 \%$ SDS, $0.125 \mathrm{M}$ Tris- $\mathrm{HCl}, \mathrm{pH} 6.8,10 \% \beta$-mercaptoethanol, $\mathrm{pH}$ adjusted to 6.8). Ten $\mu \mathrm{g}$ of the clear liquid lysate was loaded on SDS-PAGE gels for protein separation, followed by immunoblotting with the 9E10 monoclonal anti-myc antibody. 


\section{References for Methods}

30. Rischer, H. et al. Gene-to-metabolite networks for terpenoid indole alkaloid biosynthesis in Catharanthus roseus cells. Proc. Natl. Acad. Sci. USA 103, 5614-5619 (2006).

31. Hellemans, J., Mortier, G., De Paepe, A., Speleman, F. \& Vandesompele, J. qBase relative quantification framework and software for management and automated analysis of real-time quantitative PCR data. Genome Biol. 8, R19 (2007).

32. Karimi, M., Inzé, D. \& Depicker, A. GATEWAY ${ }^{\text {тм }}$ vectors for Agrobacterium-mediated plant transformation. Trends Plant Sci. 7, 193-195 (2002).

33. Kevei, Z. et al. 3-Hydroxy-3-methylglutaryl coenzyme A reductase1 interacts with NORK and is crucial for nodulation in Medicago truncatula. Plant Cell 19, 3974-3989 (2007).

34. Underwood, B. A., Vanderhaeghen, R., Whitford, R., Town, C. D. \& Hilson, P. Simultaneous high-throughput recombinational cloning of open reading frames in closed and open configurations. Plant Biotechnol. J. 4, 317-324 (2006).

35. Young, N. D. et al. The Medicago genome provides insight into the evolution of rhizobial symbioses. Nature 480, 520-524 (2011).

36. Alberti, S., Gitler, A. D. \& Lindquist, S. A suite of Gateway ${ }^{\circledR}$ cloning vectors for highthroughput genetic analysis in Saccharomyces cerevisiae. Yeast 24, 913-919 (2007).

37. Van Leene, J. et al. A tandem affinity purification-based technology platform to study the cell cycle interactome in Arabidopsis thaliana. Mol. Cell. Proteomics 6, 1226-1238 (2007).

38. Tamura, K., Dudley, J., Nei, M. \& Kumar, S. MEGA4: Molecular Evolutionary Genetics Analysis (MEGA) software version 4.0. Mol. Biol. Evol. 24, 1596-1599 (2007).

39. Van Damme, D. et al. Somatic cytokinesis and pollen maturation in Arabidopsis depend on TPLATE, which has domains similar to coat proteins. Plant Cell 18, 3502-3518 (2006).

40. Smith, C. A., Want E. J., O’Maille, G., Abagyan R. \& Siuzdak G. XCMS: processing mass spectrometry data for metabolite profiling using nonlinear peak alignment, matching, and identification. Anal. Chem. 78, 779-787 (2006).

41. Morreel, K. et al. Genetical metabolomics of flavonoid biosynthesis in Populus: a case study. Plant J. 47, 224-237 (2006). 
42. Morreel, K. et al. Mass spectrometry-based fragmentation as an identification tool in lignomics. Anal. Chem. 82, 8095-8105 (2010).

43. Oleszek, W. et al. Isolation and identification of alfalfa (Medicago sativa L.) root saponins: their activity in relation to a fungal bioassay. J. Agric. Food Chem. 38, 18101817 (1990).

44. Tava, A. et al. Triterpenoid glycosides from leaves of Medicago arborea L. J. Agric. Food Chem. 53, 9954-9965 (2005).

45. Bialy, Z., Jurzysta, M., Mella, M. \& Tava, A. Triterpene saponins from the roots of Medicago hybrida. J. Agric. Food Chem. 54, 2520-2526 (2006).

46. Tava, A. et al. New triterpenic saponins from the aerial parts of Medicago arabica (L.) Huds. J. Agric. Food Chem. 57, 2826-2835 (2009).

47. Tava, A., Pecetti, L., Romani, M., Mella, M. \& Avato, P. Triterpenoid glycosides from the leaves of two cultivars of Medicago polymorpha L. J. Agric. Food Chem. 59, 61426149 (2011).

48. Knop, M., Finger, A., Braun, T., Hellmuth, K. \& Wolf, D. H. Der1, a novel protein specifically required for endoplasmic reticulum degradation in yeast. $E M B O J .15,753-$ 763 (1996).

49. Huh, W.-K. et al. Global analysis of protein localization in budding yeast. Nature 425, 686-691 (2003).

50. Hampton, R. Y., Gardner, R. G. \& Rine, J. Role of 26S proteasome and HRD genes in the degradation of 3-hydroxy-3-methylglutaryl-CoA reductase, an integral endoplasmic reticulum membrane protein. Mol. Biol. Cell 7, 2029-2044 (1996). 

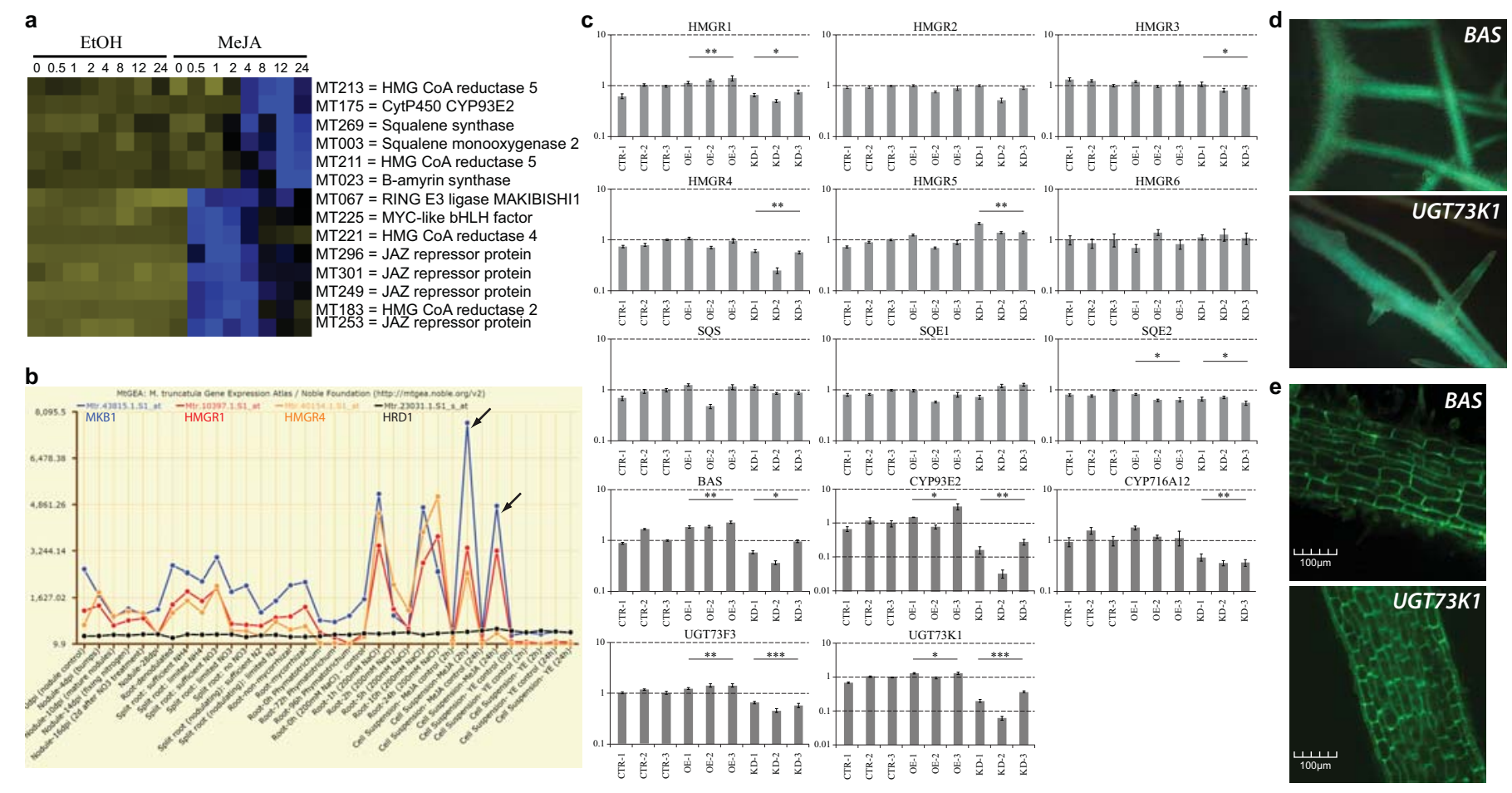


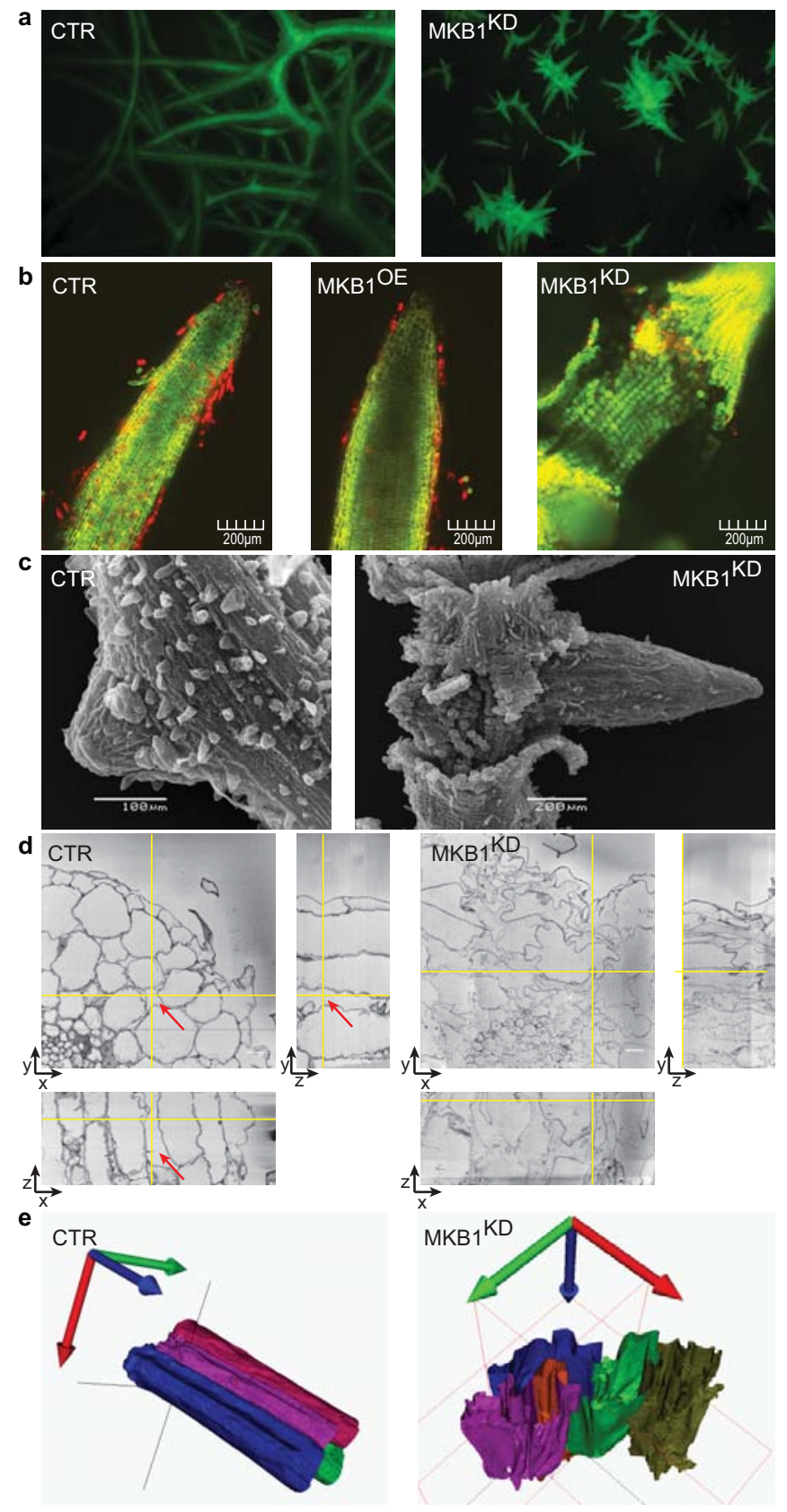




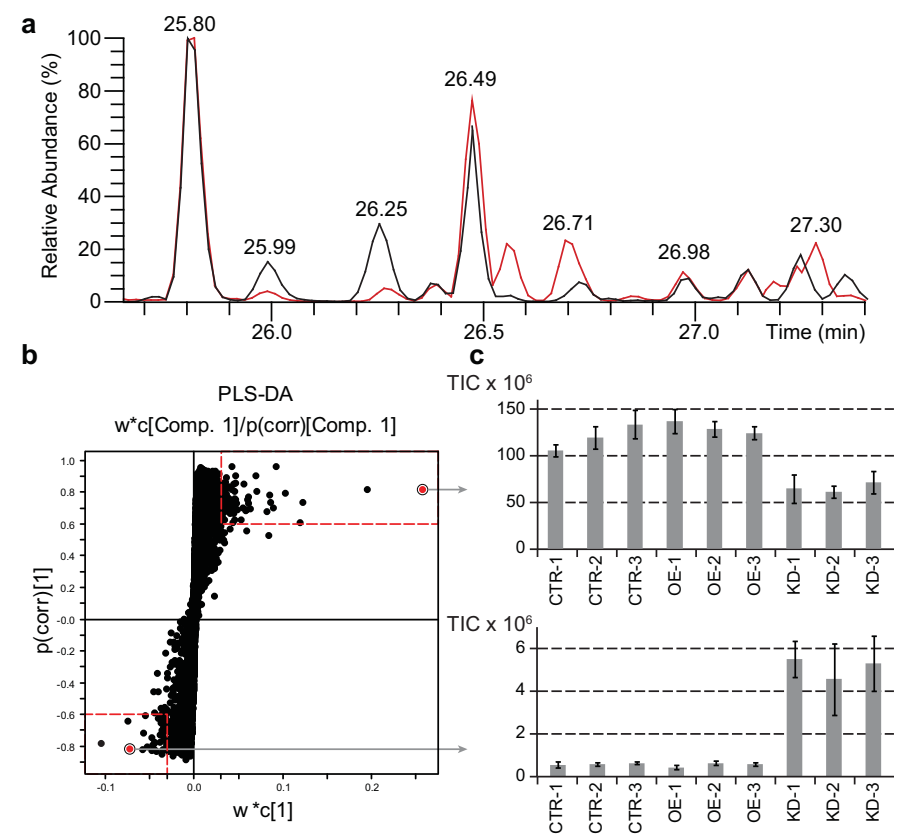



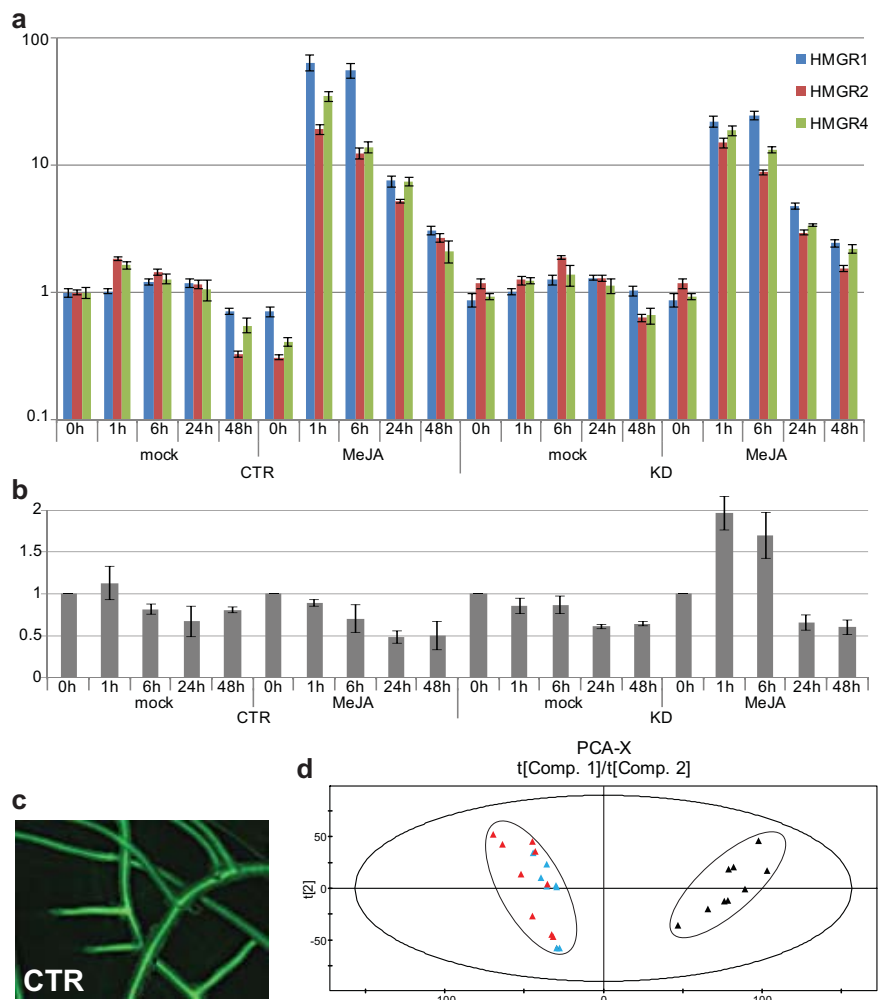
d
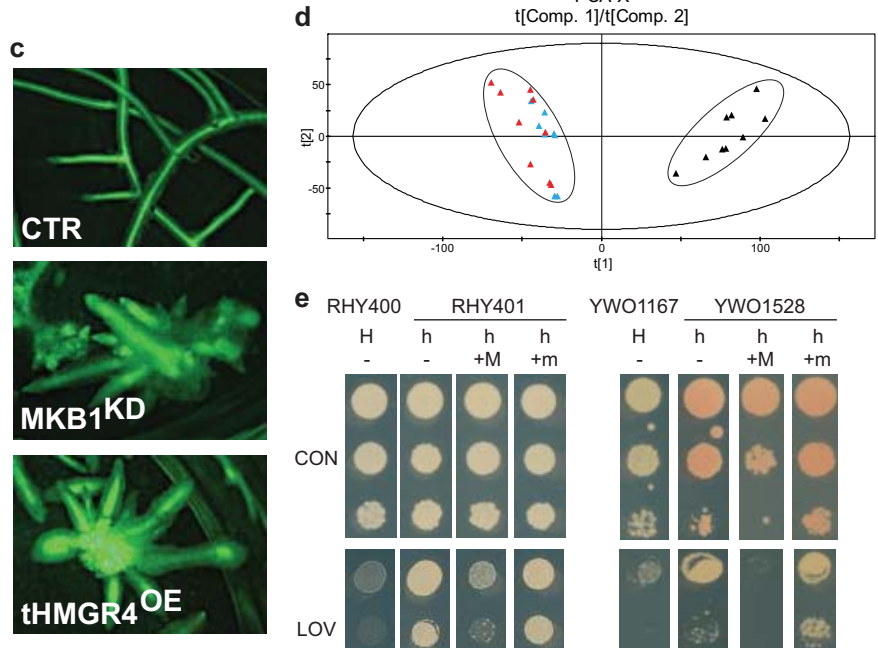

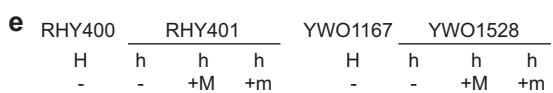

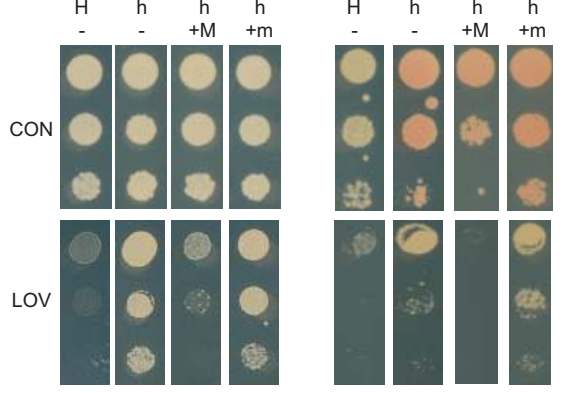

